\title{
Inversive distance
}

By H. S. M. Coxeter (Toronto)

In memory of Guido Castelnuovo, in the recurrence of the first centenary of his birth.

Summary. - Any tro non-intersecting circles have a kind of distance that is invariant for inversion, namely, the natural logarithm of the ratio of the radii (the larger to the smaller) of two concentric circles into which the given circles can be inverted. When the inversive plane is used as a conformal model for hyperbolic space [3, $p$ 266], the inversive distance between two non-intersecting circles is equal to the hyperbolic distance between the corresponding ultra-parallel planes.

\section{1. - Introduction.}

The object of this note is to encourage use of «inversive distance», which is a number determined by two non-intersecting circles in such a way as to be additive (like ordinary distance) for three circles belonging to a (non-intersecting) pencil, and invariant under the group generated by inversions. If we work over the field of complex numbers, any two circles have an angle of intersection $\delta$ or $i \delta$ (where $\delta$ is real). In the latter case the circles are nonintersecting and $\delta$ is their inversive distance; but a real treatment is more interesting.

In the final section, PoIncarte's half-plane model is used to give an extremely simple proof, kindly supplied by PAUL Szasz, of the famous formula $\Pi(x)=2$ aretan $e^{-x}$ for the angle of parallelism in hyperbolic geometry.

\section{2. - The real inversive plane.}

The inversive plane (or MöBrus plane) may be approached in various ways: (1) axiomatically, as by PIERI [8] and VAN DER WAERDEN [12], (2) as the geometry of circles on a sphere, without assigning any special role to "great» circles, $(3$; by stereographic projection, as the Euclidean plane extended by the postulation of a single point at infinity, such that lines are regarded as circles through this special point and parallel lines are regarded as circles having contact there, and (4) as the geometry of complex numbers, $x+i y$ representing the Cartesian point $(x, y)$ and $\infty$ representing the point at infinity [9]. The present treatment uses chiefily the aspects (1) and (3).

In the axiomatic approach, the primitive concepts are point, circle, and the relation of incidence. Any three distinct points $A, B, C$ determine a unique circle $A B C$, and two circles are said to be intersecting, tangent, or non-inter. 
secting according as the number of their common points is 2,1 , or 0 .

Four distinct points $A, B, C, D$ may have the property that every circle through $A$ and $B$ has at least two common points with every circle through $O$ and $D$; in this case we say that the first two separate the last two, and write $A B / / C D$. This relation implies that the four points are concyclic. For, using aspect (3), if $A, B, C, D$ are neither concyclic nor collinear, a suitable inversion allows us to regard $A B C$ as a triangle and $D$ as the point at infinity. Then the line through $C$ parallel to $A B$ is a "circle" through $C$ and $D$ which fails to meet a circle through $A$ and $B$ of sufficiently large radius. The relation $A B / / C D$ is easily seen to satisfy the axioms of VAILATI [11] which yield the familiar properties of cyclic order and sense.

Two circles $A B C$ and $B C D$ are said to be orthogonal if there exist two circles tangent to $B C D$ at $B$ and $C$ (respectively) and tangent to each other at $A[4$, p. 175]. When this happens, there are also two circles tangent to $A B C$ at $B$ and $C$ (respectively) and tangent to each other at $D$ (so that the relation of orthogonality is symmetric).

Two distinct circles $\alpha$ and $\beta$ determine a pencil of (coaxal) circles orthogonal to both. If $\gamma$ and $\delta$ are two of them, the pencil of circles orthogonal to $\gamma$ and $\delta$ includes $\alpha$ and $\beta$, and is naturally called the pencil $\alpha \beta$.

Two points $P$ and $P^{\prime}$ are said to be inverse (of each other) in a circle $\omega$ if $\omega$ is orthogonal to two distinct circles $\alpha$ and $\beta$ through $P$ and $P^{\prime}$. When this happens, $\omega$ is orthogonal to every member of the pencil $\alpha \beta$. Each point on $\omega$ is said to be its own inverse (and the centre of $\omega$ is the inverse of the point at infinity).

\section{3. - Mid-circles.}

Returning to the Euclidean aspect of the inversive plane, we can prove in various ways that the inverse of a circle is a circle, including a line as a special case. It follows that two intersecting, tangent or non-intersecting circles can be inverted into two intersecting lines, two parallel lines or two concentric circles, respectively. When a circle is inverted into a line, inversion in the circle is transformed into reflection in the line.

Given any two distinct circles $\alpha$ and $\beta$, consider the locus of a point $P$ such that two circles, tangent to both $\alpha$ and $\beta$, are tangent to each other at $P$. Inversion shows that this locus consists either of two orthogonal circles or of a single circle. More precisely, if $\alpha$ and $\beta$ intersect, we have two circles bisecting their supplementary angles of intersection; if $\alpha$ and $\beta$ are tangent, we have one circle tangent to both at the same point; and if $\alpha$ and $\beta$ are non-intersecting we have one circle, again belonging to the pencil $\alpha \beta$.

Clearly, inversion in such a circle interchanges $\alpha$ and $\beta$. The classical namc circle of antisimilitude $[1$, p. 28] is conveniently abbreviated to mid-circle. 
Thus any two distinct circles have one or two mid-circles. In particular, the radical axis of any two congruent circles is a mid-circle.

By repeated applications of the mid-circle construction, we can derive, from $\alpha$ and $\beta$, infinitely many members of the pencil $\alpha \beta$. A natural limiting process then yields the very familiar measure of angle between two intersecting circles, and a less familiar (but still inversively invariant) measure of distance between two tangent or non-intersecting circles. In the case of tangent circles this is uninteresting because the unit of measurement has no geometric significance. But two non-intersecting circles can be inverted into concentric circles of radii $a$ and $b$ where $a>b$, and we can choose such a unit that their «distance» is

$$
\log \frac{a}{b}
$$

for, if three circles of a non-intersecting pencil are inverted into concentric circles whose radii satisfy $a>b>c$, we have the proper additive relation

$$
\log \frac{a}{b}+\log \frac{b}{c}=\log \frac{a}{c}
$$

Accordingly, we define the inversive distance between any two non-intersecting circles to be the logarithm of the ratio of the radii (larger to smaller) of two concentric circles into which the given circles can be inverted. (Notice that the unit of measurement is the inversive distance between concentric circles of radii 1 and e.)

\section{4. - Inversive distance in terms of ordinary distance and radii.}

Consider, in the Euclidean plane, two oircles of radii $a$ and $b$ such that the distance between their centres is $c$. If each of $a, b, c$ is less than the sum of the other two, the circles are easily seen to intersect at two supplementary angles whose cosines are

$$
\pm \frac{a^{2}+b^{2}-c^{2}}{2 a b}
$$

As a limiting case of two such circles we can consider a line, and a circle of radius $b$ whose centre is distant $d$ from the line, so that, if $d<b$, they intersect at angles whose cosines are

$$
\pm \frac{d}{b}
$$

As a first step towards analogous results in cases of non-intersection, 
let us express inversive distance in terms of cross ratio, which is, of course, an inversive invariant $[5$, p. 87$]$.

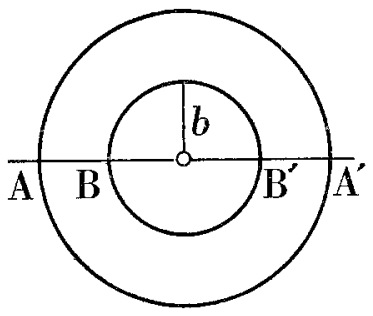

Fig. 1

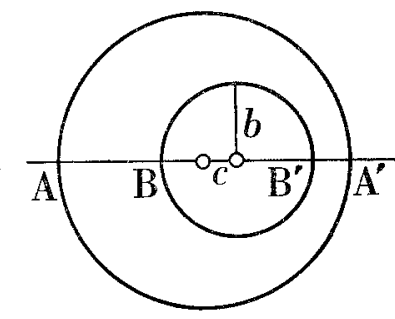

Fig. 2

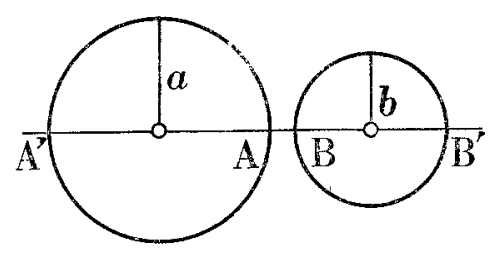

Fig. 3

Let two concentric circles, whose radii satisfy $a>b$, meet a common diameter in point pairs $A A^{\prime}, B B^{\prime}$ with $A B^{\prime} / / A^{\prime} B$, as in Figure 1 . In terms of the inversive distance

$$
\delta=\log \frac{a}{b},
$$

we find the cross ratio

$$
\begin{aligned}
\left(A A^{\prime}, B B^{\prime}\right) & =\frac{A B \times A^{\prime} B^{\prime}}{A B^{\prime} \times A^{\prime} B}=\left(\frac{A B}{A B^{\prime}}\right)^{2}=\left(\frac{a-b}{a+b}\right)^{2} \\
& =\left(\frac{e^{\delta}-1}{e^{\delta}+1}\right)^{2}=\tanh ^{2} \frac{\delta}{2}=\frac{\cosh \delta-1}{\cosh \delta+1} .
\end{aligned}
$$

If these circles arise by inversion from two non-intersecting circles whose centres are at Euclidean distance $c$, it is convenient to use the same letters $a$ and $b$ for the radii of the latter circles, and $A, A^{\prime}, B, B^{\prime}$ for the points where they cut their common diameter (with $A B^{\prime} / / A^{\prime} B$, as before). Since separation, cross ratio and inversive distance are invariant, we still have

$$
\left(A A^{\prime}, B B^{\prime}\right)=\frac{\cosh \delta-1}{\cosh \delta+1}
$$

but now we must express this cross ratio (and hence $\delta$ ) in terms of the new $a$ and $b$ along with $c$. If $a-b>c$, as in Figure 2, we have

$$
\frac{A B \times A^{\prime} B^{\prime}}{A B^{\prime} \times A^{\prime} B}=\frac{(a+c-b)(a-c-b)}{(a+c+b)(a-c+b)}=\frac{(a-b)^{2}-c^{2}}{(a+b)^{2}-c^{2}}=\frac{a^{2}+b^{2}-c^{2}-2 a b}{a^{2}+b^{2}-c^{2}+2 a b}
$$

and therefore

$$
\cosh \delta=\frac{a^{2}+b^{2}-c^{2}}{2 a b}
$$


but if $a+b<c$, as in Figure 3 ,

$$
\frac{A B \times A^{\prime} B^{\prime}}{A B^{\prime} \times A^{\prime} B}=\frac{(c-a-b)(c+a+b)}{(c-a+b)(c+a-b)}=\frac{c^{2}-(a+b)^{2}}{c^{2}-(a-b)^{2}}=\frac{c^{2}-a^{2}-b^{2}-2 a b}{c^{2}-a^{2}-b^{2}+2 a b}
$$

and therefore

$$
\cosh \delta=\frac{c^{2}-a^{2}-b^{2}}{2 a b}
$$

In either case,

$$
\cosh \delta=\left|\frac{a^{2}+b^{2}-c^{2}}{2 a b}\right|
$$

In the former case, by regarding the two circles as sections of two cylnn. ders, we can deduce an interesting connection with electrostatics. According to JEANs [7, p. 296, Ex. 78], (28) ${ }^{-1}$ is the capacity per unit length of a condenser formed by two right cylinders, one inside the other, with parallel axes, based on circles at inversive distance $\delta$.

Of course, the mid-circle of two non-intersecting circles bisects their inversive distance. Accordingly it is amusing to notice that, if the distance between the centres of two unit circles is $2(\sqrt{3}+1)$, another unit circle lying midway between them bisects their inversive distance although, not being coaxal with them, it is certainly not their mid-circle.

We see from 4.2 that, when $b$ tends to zero, $\delta$ tends to infinity. By setting $c=a \pm b$, we see that tangent circles may properly be regarded as having inversive distance zero. As another limiting case we can consider a line and a sircle of radius $b$ whose centre is distant $d$ from the line. The cross ratio reduces to an ordinary ratio so that, if $d>b$, the inversive distance between the line and the circle is $\delta$ where

$$
4.21 \quad \cosh \delta=\frac{d}{b} \text {. }
$$

\section{5. - Inversive distance in terms of Steiner's porism.}

Every rational number $p>2$ determines a regular polygon $\{p\}$ whose sides subtend angles $2 \pi / p$ at its centre [5, pp. 102-103]. If $p$ is an integer this is an ordinary $n$-gon, where $n=p$; otherwise it is a star $n$-gon, where $n$ is the numerator of the rational number $p$. If half the length of a side is used as radius, circles centred at the vertices are successively tangent and form a "ring» of $n$ circles all tangent to two concentric circles. (In Figure 4, $n=p=7$ ). If $a$ (the larger) and $b$ are the radii of these two concentric circles, we easily see that the polygon has side $a-b$, circumradius $(a+b) / 2$ and 


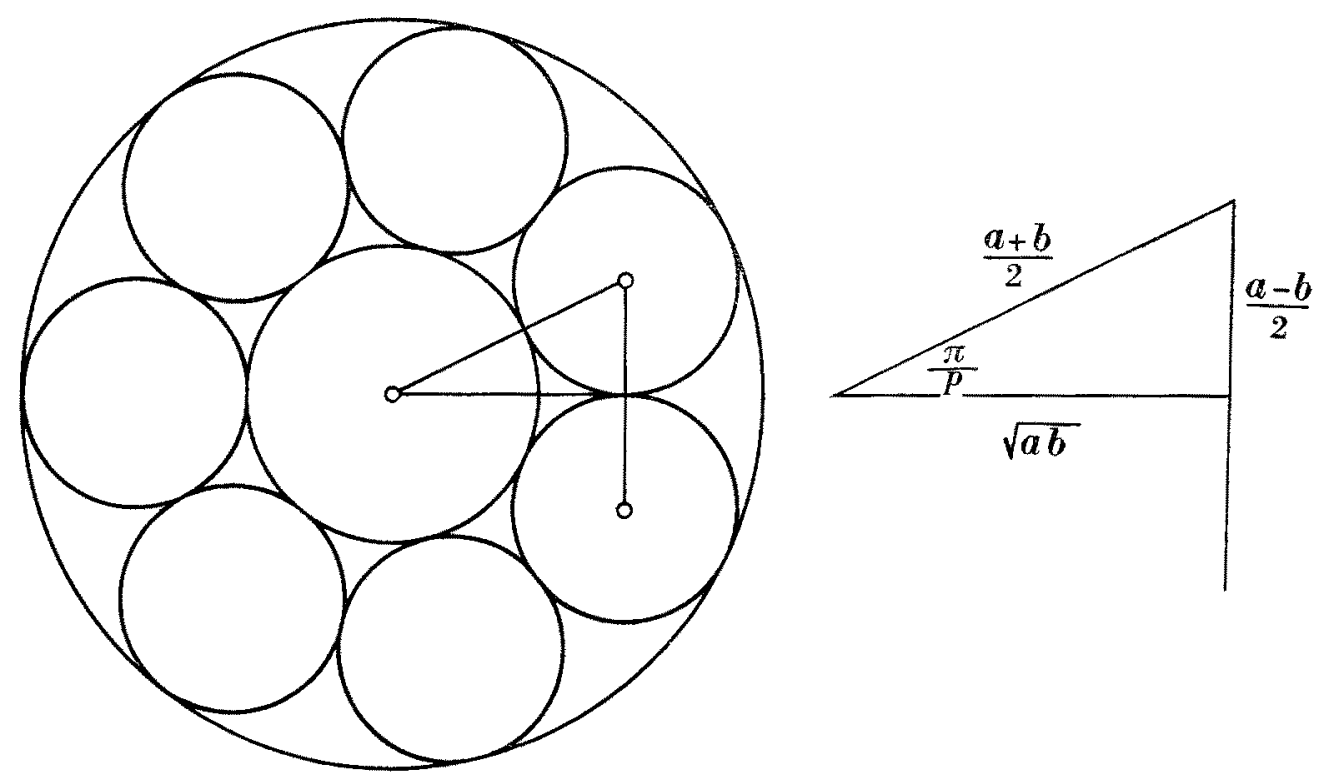

Fig. 4

inradius $V \overline{a b}$. Of course, the incircle is the mid-circle between the circles of radii $a$ and $b$. If $\delta$ is their inversive distance, we have

$$
\begin{gathered}
e^{\delta}=a / b, \\
\sin \frac{\pi}{p}=\frac{a-b}{a+b}=\frac{e^{\delta}-1}{e^{\delta}+1}=\tanh \frac{\delta}{2}, \\
\tan \frac{\pi}{2 p}=\csc \frac{\pi}{p}-\cot \frac{\pi}{p}=\operatorname{coth} \frac{\delta}{2}-\operatorname{csch} \frac{\delta}{2}=\tanh \frac{\delta}{4} \\
\sec \frac{\pi}{p}+\tan \frac{\pi}{p}=\cosh \frac{\delta}{2}+\sinh \frac{\delta}{2}=e^{\delta / 2},
\end{gathered}
$$

5.1

$$
\delta=2 \log \left(\sec \frac{\pi}{p}+\tan \frac{\pi}{p}\right)=2 \log \tan \left(\frac{\pi}{4}+\frac{\pi}{2 p}\right) .
$$

After applying any inversion, we still have a ring of $n$ successively tan. gent circles, all tangent to two non-intersecting circles whose inversive distance $\delta$ is given by 5.1 , and we recall the observation of Sremen $[10$, p. 43] that any eircle tangent to these two will serve as the first member of such a ring. Conversely, if $\delta$ is the inversive distance between two given non-intersecting circles, a sequence of successively tangent circles touching both can be ap- 
proximated as closely as we please by a ring whose $p$ is a rational approximation to

$$
\pi / \arcsin \left(\tanh \frac{\delta}{2}\right)
$$

In particular, any three circles tangent to one another at distinct points are tangent to two non-intersectiug circles [6, p. 216, Ex. 29] whose inversive distance $\delta$ is given by

$$
\cosh \frac{\delta}{2}=\sec \frac{\pi}{3}=2, \quad \delta=2 \log (2+\sqrt{3}) .
$$

In other words, this is the inversive distance between the two SoDDY circles of any triangle [2, pp. 13-14].

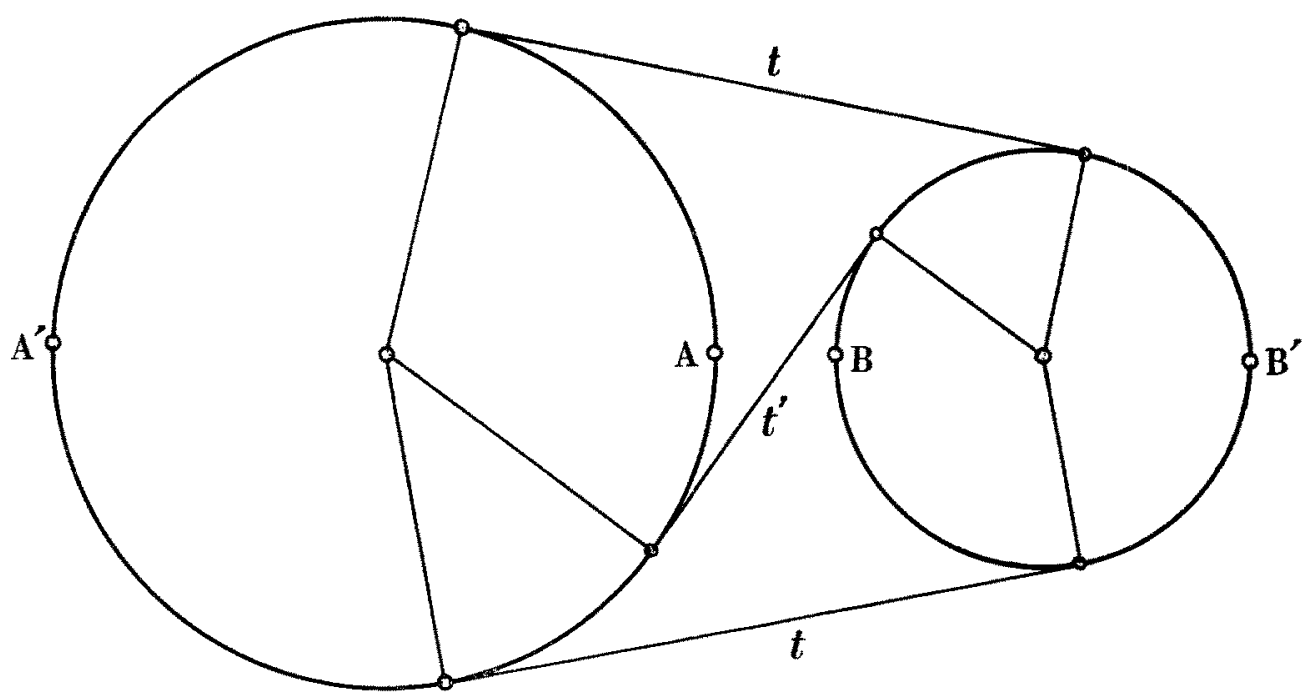

Wig. 5

\section{6. - Inversive distance in terms of common tangents.}

If $a+b<c$, as in Figure 3, the circles have four common tangents: two of length $t$ (say), belonging to the convex hull of the pair of circles, and two (crossing each other) of a shorter length $t^{\prime}$. (See Figure 5.) An easy calculation shows that their squares are equal to

$$
c^{2}-(a \mp b)^{2}
$$


thus

$$
\left(\frac{t^{\prime}}{t}\right)^{2}=\frac{c^{2}-(a+b)^{2}}{c^{2}-(a-b)^{2}}=\left(A A^{\prime}, B B^{\prime}\right)=\tanh ^{2} \frac{\delta}{2}
$$

and the inversive distance $\delta$ is giveu by

6.1

$$
\tanh \frac{\delta}{2}=\frac{t}{t}
$$

Unhappily, this simple formula is not available when one circle encloses the other (as in Figure 2) unless we agree to embed the real plane in a complex plane and allow the common tongents to have pure-imaginary lengths.

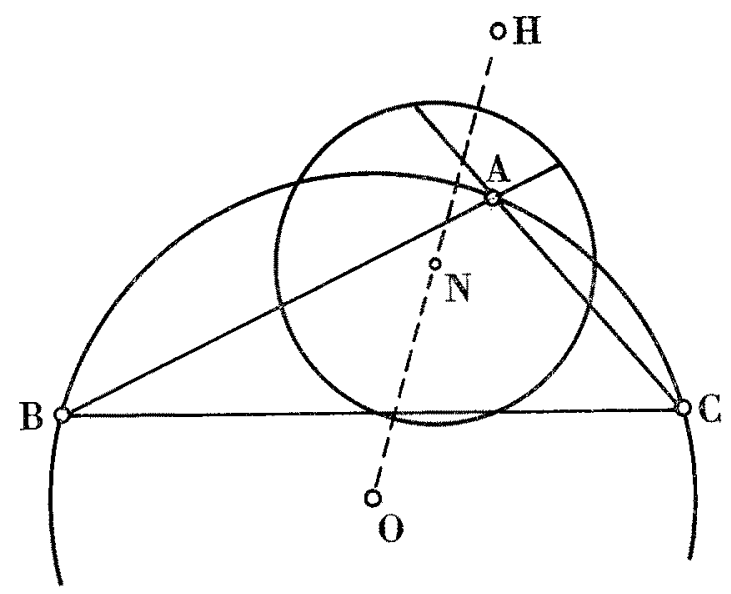

Fig. 6

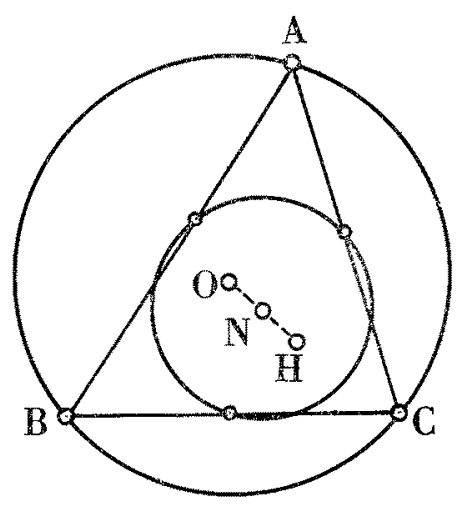

Fig. 7

\section{7. - Circles related to a triangle.}

We recall that the vertices of any triangle $A B C$ lie on the circumcircle, of radius $R$ and centre $O$, while the midpoints of the sides lie on the ninepoint circle (EULER-Feuerbach circle), of radius $R / 2$, whose centre $N$ is midway between te circumcentre $O$ and the orthocentre $H[2, \mathrm{pp}, 17,71]$. Since

$$
O H^{2}=R^{2}(1-8 \cos A \cos B \cos C)
$$

$[6$, p. 119], we can find the angle or distance betwee these two circles by substituting

$$
R \text { for } a, R / 2 \text { for } b \text {, and } O H / 2 \text { for } c
$$

in 4.1 or 4.2 . The results are as follows:

If the triangle $A B C$ is obtuse-angled, as in Figure 6, the circumcircle cuts 
the nine-point circle at an angle $\delta$ such that

$$
\sin \frac{\delta}{2}=\sqrt{-\cos A \cos B \cos C}
$$

But if the triangle is acute-angled, as in Figure 7 , the circumoircle encloses the nine-point circle, and their inversive distance $\delta$ iu given by

$$
\sinh \frac{\delta}{2}=\sqrt{\cos A \cos B \cos C} .
$$

Similarly, by substituting

$$
R \text { for } a, \quad r \text { for } b, \quad \text { and } R^{2}-2 R r \text { for } c^{2}
$$

in 4.2, we find that the circumeircle and the incircle (of radius $r$ ) have inversive distance $\delta$ such that

$$
\sinh \frac{\delta}{2}=\frac{1}{2} \sqrt{\frac{r}{R}}
$$

In both these last two cases, the inversive distance attains its maximum, $\delta=\log 2$, when the triangle is equilateral.

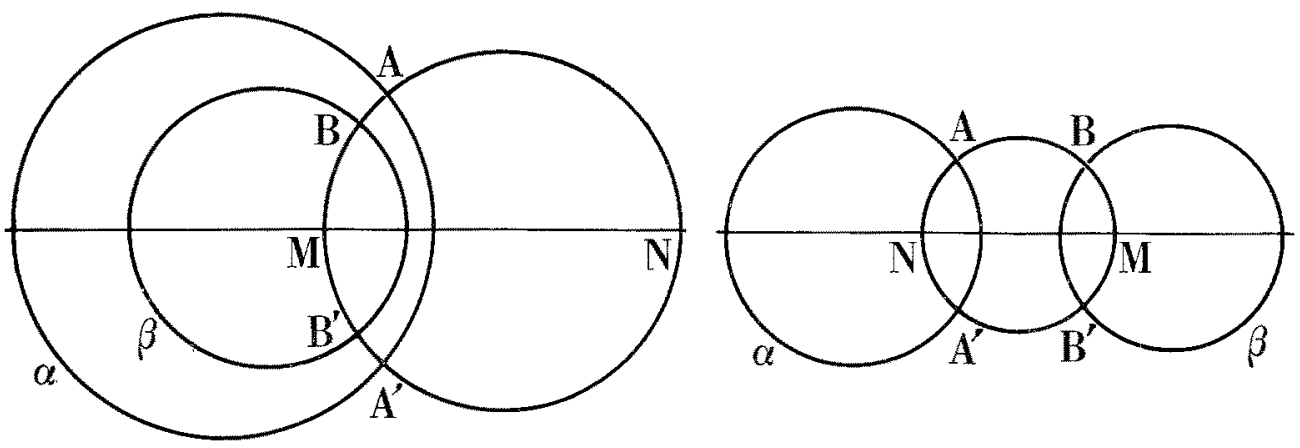

Fig. 8

\section{8. - Hyperbolic geometry.}

In Poincaré's half-plane model of the hyperbolic plane [5, p. 88 ; 3 , p. 263], angles are represented faithfully but distances are expressed in terms of cross ratios, as follows. To find the hyperbolic distance $\delta$ between two points repre. send by $A$ and $B$, we draw the circle through $A$ and $B$ orthogonal to the peripheral line (representing the absolute). Then we let $A^{\prime}$ and $B^{\prime}$ (on this circle) be the images of $A$ and $B$ by reflection in the line, let $M$ and $N$ be 
the points of intersection of the circle and the line (as in Figure 8), and use either of the two equivalent formulae

$$
8.1 \quad \delta=\log (A B, M N), \quad \tanh \frac{\delta}{2}=\left(A A^{\prime}, B B^{\prime}\right)^{\frac{1}{2}} .
$$

Let $\alpha$ and $\beta$ denote the circles through the point pairs $A A^{\prime}$ and $B B^{\prime}$ (respectively) orthogonal to the circle $A M N$. These two circles represent ultraparallel lines having a common perpendicular represented by $A B$. The distance between the lines is naturally defined to be the distance along their common perpendicular, namely $\hat{o}$. It follows that the hyperbolic distance between two ultraparallel lines is equal to the inversive distance between their represen. tative circles. For, the formula

$$
\left(A A^{\prime}, B B^{\prime}\right)=\tanh ^{2} \frac{\delta}{2}
$$

which appeared in $\S 4$, expresses the inversive distance between the two circles in terms of their points of intersection with any circle orthogonal to both. In Figures 2 and 3 we used the line of centres, but any other eircle through $M$ and $N$ (as in Figure 8) must yield the same value for the cross ratio.

In particular, $\alpha$ and $\beta$ may be concentric; then $N$ is the point at infinity, the cross ratios reduce to simple ratios, and we have

$$
\delta=\log \frac{A M}{B M}, \quad \tanh \frac{\delta}{2}=\frac{A B}{A B^{\prime}} .
$$
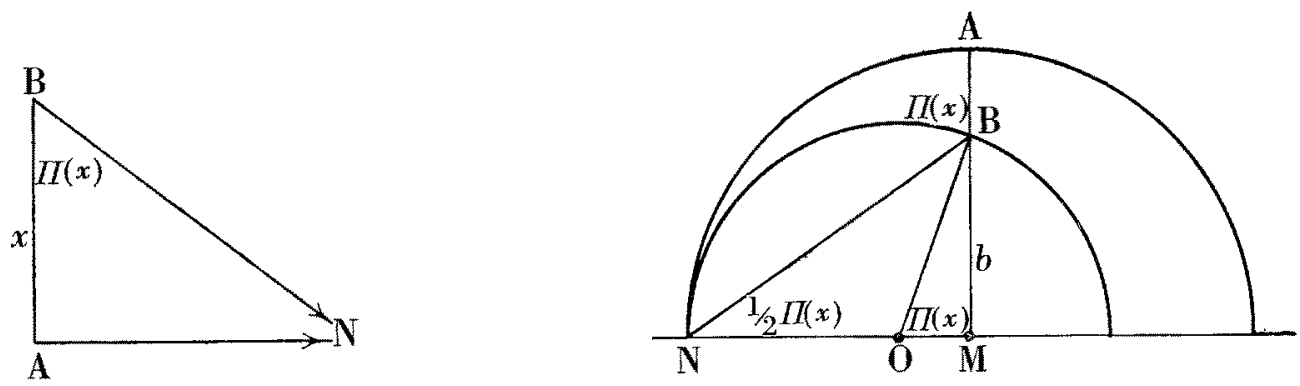

Fig. 9

\section{9. - The angle of parallelism.}

These ideas can be used to derive, in the simplest possible way, the famous formula of BOLYAX and LOBACHEVSKY for the angle of parallelism $\Pi(x)$ corresponding to a given distance $x$ in the hyperbolic plane. In Figure 9 , the segment of length $x$ is represented by the part $A B$ of a vertical segment 
$A M$ whose end $M$ lies on the peripheral line. The circle through $A$ with centre $M$ represents a line perpendicular to the segment of length $x$, and the tangent eircle through $B$ with centre $O$ (also on the peripheral line) represents a parallel line (in the sense of hyperbolic geometry).

In terms of the Euclidean distances $a=M A, b=M B$, we have, from 8.2,

$$
x=\log \frac{a}{b}
$$

Elementary properties of the Euclidean plane yield the angles

$$
M O B=\Pi(x), \quad M N B=\frac{1}{2} \Pi(x),
$$

and consequenty

$$
\cot \frac{1}{2} \Pi(x)=\frac{M N}{M B}=\frac{M A}{M B}=\frac{a}{b}=e^{x}
$$

Thus

$$
\Pi(x)=2 \arctan e^{-x}
$$

\section{REFERENCES}

[1] J. L. Coolidge, A treatise on the eircle and the sphere (Oxford, 1916).

[2] H. S. M. CoxwTer, Introduction to geometry (New York, 1961).

[3] - -, Non-Euclidean geonetry (כ̌th ed., Toronto 1965).

[4] Peter Dembowskt and D. R. Hughes, On finite inversive planes, J. Irondon Math. Soc. 40 (1965), 171-182.

[5] László FeJes Tóth, Regular figures (New York, 1964).

[6] E. W. Hobson, A treatise on plane trigonometry (6th ed., Cambridge, 1924).

[7] J. H. JaANs, The mathematical theory of electricity and magnetism (5th ed., Cambridge, $1925)$.

[8] Mario PIERI, Nuovo principiti di geometria delle inversioni, Giornale di Math. (3), 49 (1911), 49-96; 50 (1912), 106-140.

[9] Hans ScHWerdtFeger, Geometry of complex numbers (Toronto, 1962).

[10] J ACOB STEINER, Gesammelte Werke 1 (Berlin, 1882).

[11] Giovanni Vallati, Sulle relazioni di posizione tra punti d'una linea chiusa, Rivista di Mat. 5 (1895), 75-78, 183-185

[12] B. L. VAN DER WAERDEN and L. J. SMID, Eine Axiomatik der Kreisgeometrie und der Laguerregeometrie, Math. Ann. 110 (1935), 754-776. 